\title{
Generation of Unstructured Tetrahedral Meshes for Flow Past Flight Vehicles Based on Open Packages
}

\author{
Michael K. Ermakov ${ }^{1}$, Arina Kryuchkova ${ }^{1,2}$ \\ ${ }^{1}$ Ishlinsky Institute for Problems in Mechanics RAS, \\ Moscow, 119526, Russia \\ ${ }^{2}$ Moscow Institute of Thermal Technology, \\ Moscow, 127273, Russia \\ ermakov@ipmnet.ru, kryuchkova.arina.s@,gmail.com
}

\begin{abstract}
The open packages Gmsh and Salome are used for automatic generation of unstructured tetrahedral meshes in aircraft flow problems. These packages allow you to quickly and efficiently build spatial grids for geometric models defined in the OpenCascade geometric core or in STEP format. It provides mechanisms for thickening the grid, adapting the grid to the solution, generating the grid in interactive mode, command-line mode, and using the program library. In this paper, the possibilities of grid generation algorithms in terms of speed and quality of the resulting grid are investigated. Examples of creating a geometric model and generating a grid are given, as well as examples of calculations on the resulting grids.
\end{abstract}

Keywords: unstructured mesh, tetrahedral mesh, grid generation, Gmsh, Salome
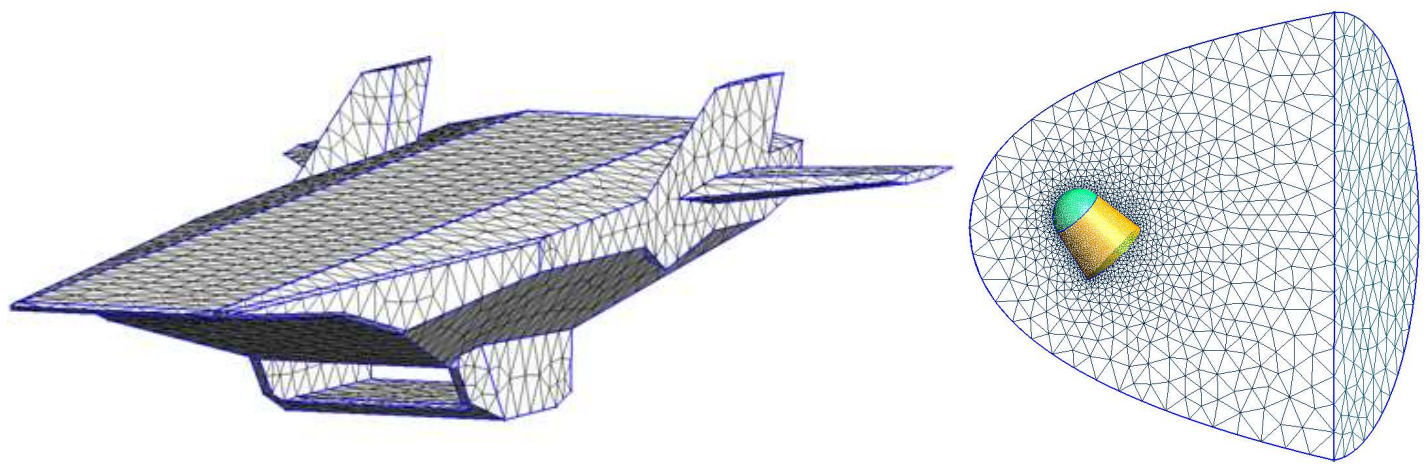

Two examples of unstructured tetrahedral grids generated automatically for the X-43 flight vehicle and a cone rounded by a sphere in a flow area. Automatic mesh generation is performed by Gmsh and Salome open packages 
УДК $519.688,533.6$

\title{
Генерация неструктурированных тетраэдральных сеток для обтекания летательных аппаратов на основе открытых пакетов
}

\author{
М. К. Ермаков ${ }^{1}$, А. С. Крючкова ${ }^{1,2}$ \\ ${ }^{1}$ Институт проблем механики им. А. Ю. Иилинского РАН, \\ Россия, Москва, 119526, просп. Вернадского 101, корп. 1 \\ ${ }^{2} \mathrm{AO}$ «Московский Институт Теплотехники», \\ Россия, Москва, 127273, Берёзовая аллея 10, корп. 14 \\ ermakov@ipmnet.ru, kryuchkova.arina.s@gmail.com
}

\begin{abstract}
Аннотация
Для автоматической генерации неструктурированных тетраэдральных сеток в задачах обтекания летательных аппаратов в работе используется открытое программное обеспечение Gmsh и Salome. Указанные пакеты позволяют быстро и качественно строить пространственные сетки для геометрических моделей, заданных в геометрическом ядре OpenCascade или в формате STEP. Обеспечиваются механизмы сгущения сетки, адаптации сетки к решению, генерация сетки в интерактивном режиме, режиме командной строки и использование библиотеки программ. В работе исследованы возможности алгоритмов генерации сетки с точки зрения быстродействия и качества получаемой сетки. Приведены примеры создания геометрической модели и генерации сетки, а также примеры расчетов на полученных сетках.
\end{abstract}

Ключевые слова: неструктурированные сетки, тетраэдральные сетки, генерация сеток, Gmsh, Salome

\section{1. Введение}

Построение пространственных сеток в автоматическом режиме для моделирования обтекания аэрокосмических летательных аппаратов (ЛА) и объектов является актуальной научно-технической задачей. При кажущейся простоте постановки задача генерации сетки является достаточно сложной и ресурсоемкой даже при использовании простейших пространственных элементов в виде тетраэдров. Используемые до недавнего времени программные средства были существенно ограничены автоматической генерацией сеток, достигающих десятков миллионов ячеек, и в быстродействии.

Программные продукты по генерации сеток часто являются составными частями программных сред, включающих системы автоматизированного проектирования и моделирования, либо представлены в виде отдельных пакетов ICEM, PointWise и других. Современные программные среды типа ANSYS являются довольно сложными и громоздкими с одной стороны, и довольно дорогими, с другой стороны. В связи с этим представляют интерес достаточно многочисленные открытые пакеты генераторы сеток Gmsh, Salome, EnGrid, Netgen, Tetgen, Ani3D [1] и входящие в OpenFOAM программы BlockMesh, PolyDualMesh и snappyHexMesh. 
Опыт работы с открытыми пакетами показывает, что они могут вполне успешно использоваться для автоматической генерации пространственных сеток для обтекания объектов сложной формы. В данной работе описаны и исследованы возможности двух достаточно распространенных пакетов Gmsh и Salome для задания геометрии и автоматической генерации пространственных тетраэдральных сеток для задач обтекания аэрокосмических объектов.

\section{2. Общее описание открытых пакетов}

\section{1. Описание Gmsh}

Программный комплекс Gmsh (версия 4.7.1) [2-4] предназначен для автоматической генерации неструктурированных поверхностных и пространственных сеток для геометрических объектов, заданных граничными поверхностями. Набор поверхностей задается как на основе собственного упрощенного геометрического ядра, так и на основе геометрического ядра и программных средств OpenCascade. Система Gmsh представляет собой интерактивную среду, включающую геометрический модуль в виде сильно упрощенной подсистемы САПР, модуль генерации сеток и модуль визуализации конечно-элементных данных. Использование развитой открытой системы OpenCascade позволяет использовать широкий круг функций геометрического ядра: широкий набор примитивов поверхностей и объемов, набор булевских операций над геометрическими объектами, параметризацию и документирование процесса построения объекта и создаваемой сетки в виде текстового скрипта, систем импорта геометрии в стандартных форматах и экспорта геометрии.

Помимо генерации поверхностных треугольных сеток пакет поддерживает преобразование треугольных поверхностных сеток в сетки с преимущественно четырехугольными элементами, а также образование на их основе призматических элементов при преобразовании выталкивания (вытягивания) геометрии и сетки. Сетки, однако, в любом случае остаются неструктурированными.

Набор алгоритмов построения сеток базируется на сочетании метода Делоне, метода продвигаемого фронта и методов разбиения и перестроения сетки. Методы характеризуются различной степенью качества построения сетки, быстродействием и степенью надежности (робастности, безотказности), обозначающей возможности создания сетки для геометрии сложного типа.

Пакет написан полностью на алгоритмическом языке $\mathrm{C}++$ и функционирует в интерактивном режиме и в пакетном режиме (командной строки) в операционных системах Windows/Linux/MacOS. Посредством использования API с подключением библиотек пакет доступен в алгоритмических языках $\mathrm{C} / \mathrm{C}++$, Fortran, Python и Julia. Пакет Gmsh развивался в течение 20 лет и продолжает активно развиваться авторами, имеет подробную документацию с примерами и поддержку разработчиков в виде форума. В настоящее время Gmsh, по мнению авторов, является одним из наиболее функциональных и производительных пакетов для генерации тетраэдральных пространственных сеток.

\section{2. Описание Salome}

Открытый программный пакет Salome (версия 9.7.1) [5, 6] развивается с 2000 года и представляет собой универсальную платформу среды для работы с CAD-моделью на протяжении её полного жизненного цикла, включая генерацию сеток, интеграцию вычисленных кодов и представление численных расчётов. В состав интегрированных кодов входят МКЭ пакет общего назначения Code-Aster и CFD пакет Code-Saturne, поддерживающий вычисления на различных типах сеток для метода конечного объема. Пакет Salome является кроссплатформенным. Пакет характеризуется интуитивно понятным интерфейсом, встроенным языком программирования пакета является Python.

Посредством Salome пользователь может импортировать, создавать, экспортировать геометрию в различных форматах (BREP, STEP и другие), создавать сетки, контролировать 
их качество. Предусмотрены встроенные инструменты, позволяющие исправлять ошибки в CAD-моделях и готовых сетках. Поддерживается создание тетраэдральных, гексаэдральных и смешанных сеток. Построение поверхностных треугольных и пространственных тетраэдральных сеток основано на методе продвигаемого фронта [7].

\section{3. Методы построения сеток}

\section{1. Построение и оптимизация сеток в Gmsh}

Используется несколько подходов к заданию локального размера создаваемой сетки. В первом подходе создается сетка, близкая к равномерной, с указанием минимального и максимального размеров сетки. Во втором подходе каждой точке (вершине) топологии геометрического объекта приписывается дополнительный параметр, определяющий локальный линейный размер сетки. На топологических элементах объекта локальный линейный размер элементов интерполируется линейной функцией. Помимо задания линейного размера сетки возможно ограничение величины шага сетки по угловому размеру элемента, определяемого кривизной поверхности. Третий подход заключается в задании дополнительных областей простой формы (шар, параллелепипед), в которых задается локальный линейный размер сетки.

После задания локального шага система последовательно, по командам пользователя, строит одномерную сетку вдоль ребер объекта (1D), двумерную сетку на поверхностях объекта (2D) и трехмерную сетку внутри объемов объекта (3D). По желанию пользователя каждая сетка в виде элементов, таких как, вершины, ребра, грани и тетраэдры с их номерами или без номеров могут визуализироваться. Также возможна визуализация отдельных элементов или их групп, относящихся к любому виду элементов топологии. После этапа генерации двумерной сетки возможно улучшение качества сетки, число итераций сглаживания задается. После этапа построения трехмерной сетки возможна оптимизация сетки с целью улучшения качества элементов.

В качестве алгоритмов построения тетраэдральной пространственной сетки используются 3-D варианты метода Делоне для алгоритмов Delaunay [8, 9] и HXT [3], метод продвигаемого фронта J. Schöberl для алгоритма Frontal [7]. Собственная оптимизация Gmsh тетраэдральных сеток построена на перестановках ребер и граней с перемещением вершин [2]. Дополнительно подключаемая библиотека оптимизации Netgen основана на сочетании локального метрического сглаживания и топологических преобразований [7].

\section{2. Построение и оптимизация сеток в Salome}

Технология создания сетки в Salome включает в себя назначение правил (именуемых в пакете гипотезами) и алгоритмов для сеток рассматриваемого и низшего порядка (например, 1D, 2D, 3D-гипотезы для создания пространственной сетки). Общий подход к заданию размеров сетки похож на первый из подходов, использующихся в Gmsh и описанных в разделе выше. Salome генерирует сетки назначенных порядков, последовательно следуя от низшего к высшему порядку разбиения. Доступно 2 типа 1D-правил, первое из них - разбиение линии (Wire Discretization) - разбивает 1D-элементы на заданное количество сегментов или на сегменты с заданным размером, второе - разбиение составной линии (Composite Side Discretization) - позволяет применить правило целиком на всё ребро геометрической грани, даже если оно состоит из нескольких линий. Генерация пространственных тетраэдральных сеток осуществляется методом продвигаемого фронта Netgen [7], реализуемой той же библиотекой, что и в пакете Gmsh. В связи с этим, результаты исследования алгоритма Frontal из Gmsh автоматически переносятся на результаты алгоритма Netgen в Salome.

Аналогично Gmsh, Salome позволяет визуализировать отдельные элементы сетки или их группы, выделенные на базе элементов геометрической топологии или иных фильтров (принадлежность к свободным границам, двойным узлам, диапазону ID элементов). Имеются 
алгоритмы сглаживания сетки, конвертации поверхностной треугольной сетки в сетку, состоящую из четырехугольников, и набор инструментов для выявления и исправления ошибок построения сетки (удаление и дублирование элементов, сшивка сеток на свободных границах).

Анализ возможностей пакетов Gmsh и Salome показал, что Gmsh имеет несколько более широкий функционал и является более простым и удобным в работе, поэтому дальнейшее исследование возможностей по генерации сеток будет проведено на его основе. К преимуществам Salome, вместе с тем, относятся его широкая распространенность, полнофункциональная CAD система и интеграция с несколькими пакетами моделирования, а также наличие дополнительных коммерческих генераторов сеток.

Следует упомянуть, что вопросы построения геометрических моделей сложных ЛА и областей их обтекания на основе промышленных САПР решались в работах $[10,11]$. Вопросы генерации сеток для этих объектов на основе метода молекулярной динамики для тетрадральных сеток и на основе электростатической аналогии для слоистых пространственных сеток рассматривались в основанных на авторских кодах работах [12, 13].

\section{4. Практика работы в Gmsh}

\section{1. Интерактивный режим}

Внешний вид системы в интерактивном режиме со стандартным горизонтальным меню приложения и вертикальным меню древовидной структуры, геометрией объекта, представленной ребрами геометрии, и поверхностной сетки ЛА X-43 представлен на рис. 1. Стандартное горизонтальное меню позволяет изменять многочисленные параметры системы в открываемых диалоговых панелях. Благодаря горизонтальному меню возможен выбор параметров сгущения сетки, алгоритмов построения поверхностной и пространственной сеток, параметрам визуализации элементов сетки, данным по использованию ресурсов для построения сетки и другим параметрам. Находящееся в левой части окна приложения вертикальное меню представляет собой средства для взаимодействия с моделью, в частности, включает в себя набор выполняемых действий (команд). В графическом окне системы представлены ребра геометрии объекта и поверхностная сетка, которая по умолчанию рисуется разным цветом на каждой поверхности. В целях наглядности сетка выбрана с малым количеством треугольников на поверхности и без области обтекания, которая строится вокруг ЛА.

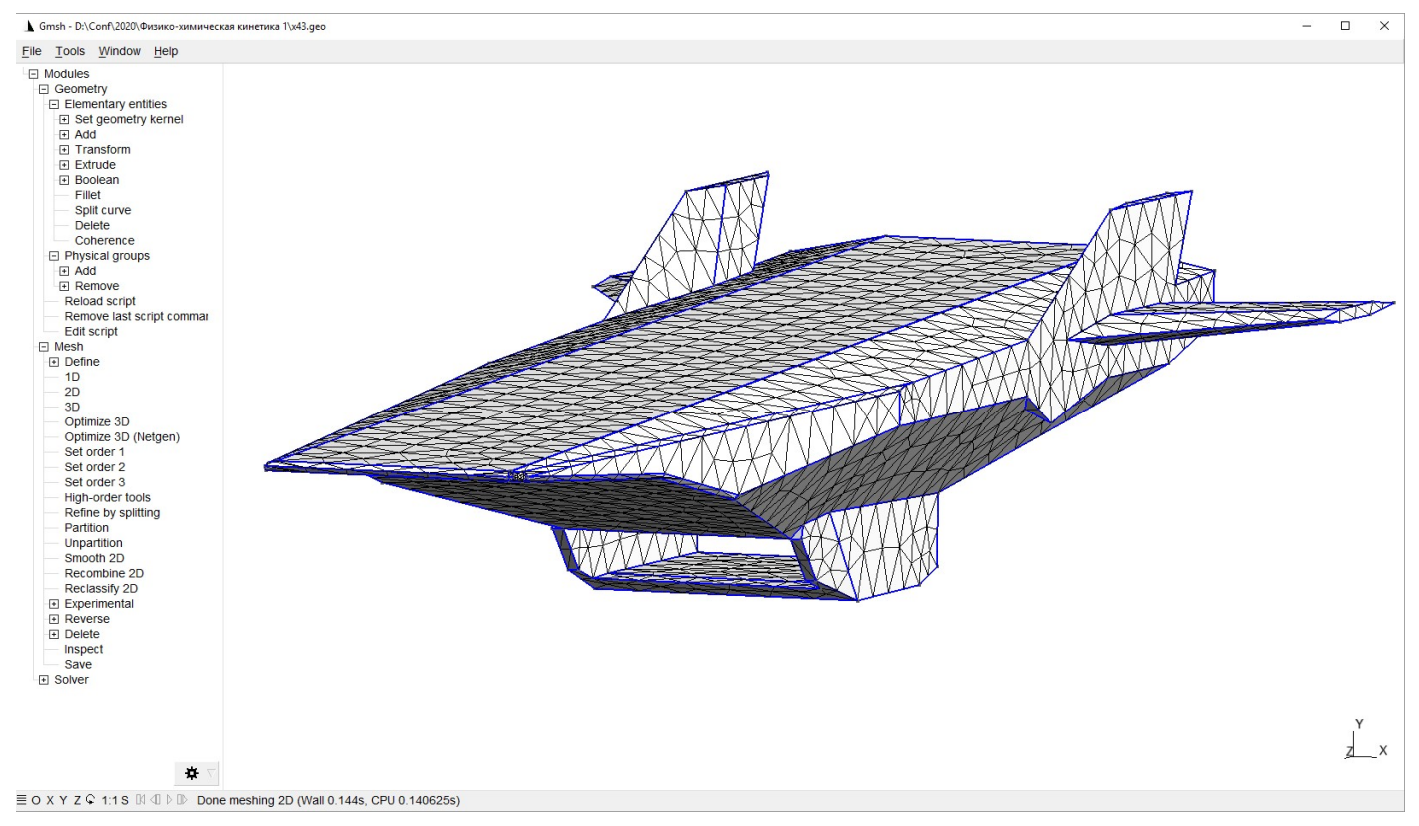

Рис. 1. Вид оболочки пакета Gmsh с развернутым древовидным меню (слева) и изображением поверхностной треугольной сетки летательного аппарата X-43 
В строке статуса обозначены выполненная операция - построение двумерной сетки и время выполнения операции. Для контроля действий в процессе выполнения операций возможно открытие окна сообщений, в которое выводится информация о ходе генерации сетки для элементов объекта.

\section{2. Пакетный режим (режим командной строки)}

Для применения пакета с минимальным использованием дополнительных ресурсов и в пакетном режиме имеется не интерактивный режим работы в командной строке. Для его использования параметры алгоритмов и выполняемые команды задаются в загружаемом текстовом скрипте с расширением geо и в параметрах командной строки вызова пакета Gmsh. B данном режиме построение и оптимизация сеток происходит полностью автономно.

\section{3. Создание параметрической геометрической модели и генерация сетки}

Система Gmsh имеет подсистему CAD, которая позволяет выполнять базовые операции по созданию и модификации геометрических объектов, используя собственный язык для таких операций. В качестве геометрического ядра при этом используется либо собственное геометрическое ядро, либо открытое геометрическое ядро OpenCascade. Последнее необходимо применять при наличии операций со сплайнами или булевых операций над телами. Существенным преимуществом использования языка для сценариев геометрических операций и построения сетки является возможность хранения и редактирования такого сценария в текстовом виде. Благодаря такому языку возможно создание простых геометрических объектов, поворот объекта под углом атаки и создание области обтекания для выбранных параметров набегающего потока. В качестве примера рассмотрим обтекание конуса, затупленного по сфере. В силу симметрии задачи задается только половина конуса и области течения. Ниже будут приведены пронумерованные операторы примера текстового скрипта, описывающего полный цикл по созданию модели и генерации сетки.

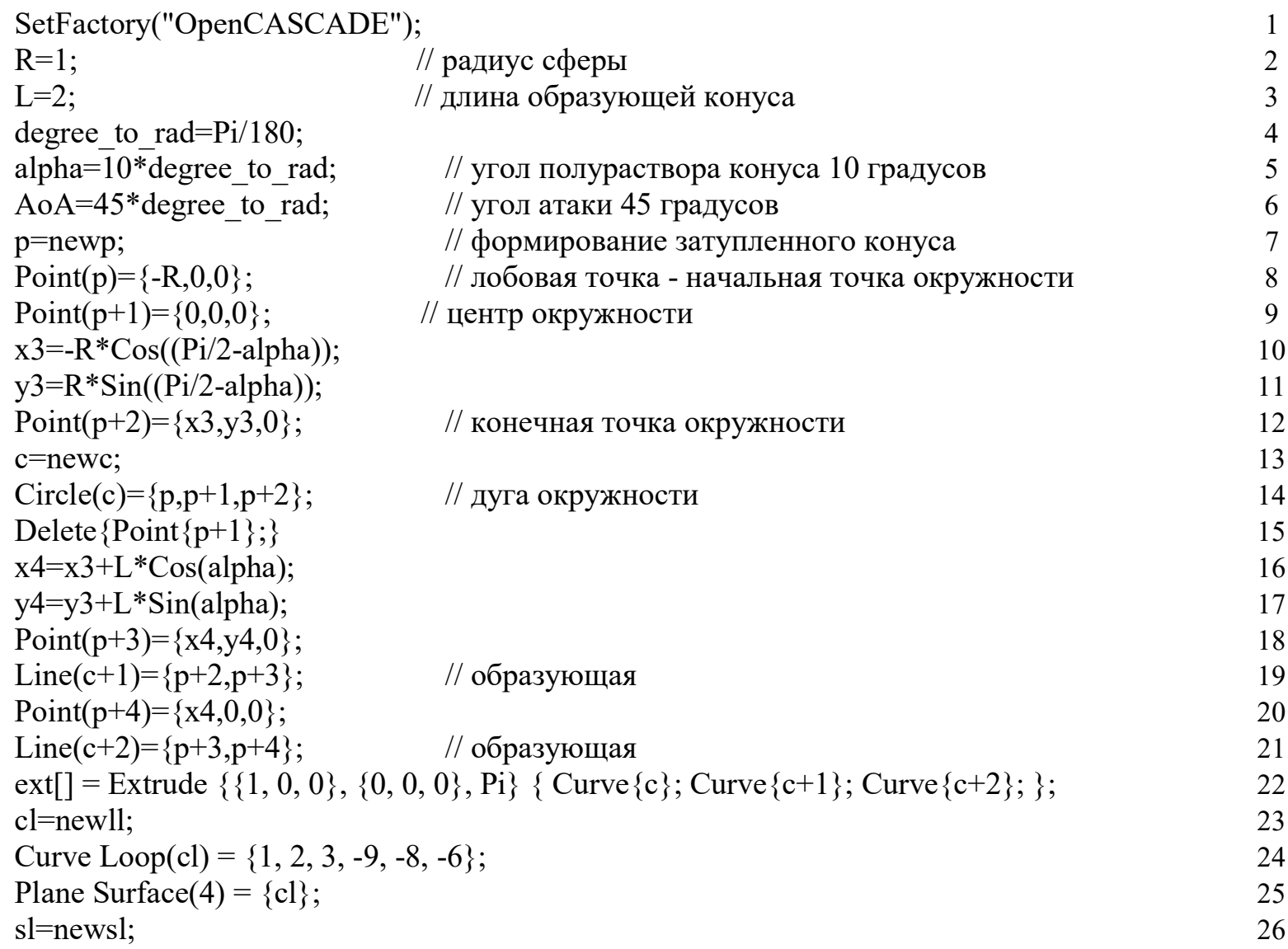


Surface Loop $(\mathrm{sl})=\{1,2,3,4\} ; \quad 27$

Delete $\{$ Surface $\{1,2\} ;\} \quad 28$

Volume $(1)=\{\mathrm{sl}\}$;

Rotate $\{\{0,0,1\},\{(\mathrm{L}-\mathrm{R}) / 1.5,0,0\},-$ AoA $\}$ Volume $\{1\} ;\}$

$\mathrm{x} 0=-4 * \mathrm{R} ; \quad$ // формирование области течения 31

$\mathrm{x} 1=10 * \mathrm{R} ;$

$\mathrm{H}=8 * \mathrm{R} ;$

$\mathrm{p}=$ newp; $\quad 34$

Point $(\mathrm{p})=\{\mathrm{x} 0,0,0\} ; \quad 35$

Point $(\mathrm{p}+1)=\left\{\mathrm{x} 0,0.66^{*} \mathrm{H}, 0\right\} ;$

Point $(\mathrm{p}+2)=\{\mathrm{x} 1, \mathrm{H}, 0\} ; \quad 37$

Point $(\mathrm{p}+3)=\{\mathrm{x} 1,0,0\} ;$

$\mathrm{c}=$ newc; $\quad 39$

BSpline $(\mathrm{c})=\{\mathrm{p}, \mathrm{p}+1, \mathrm{p}+2\} ; \quad 40$

Delete $\{$ Point $\{p+1\} ;\}$

Line $(\mathrm{c}+1)=\{\mathrm{p}+2, \mathrm{p}+3\} ;$

$\operatorname{ext}[]=$ Extrude $\{\{1,0,0\},\{0,0,0\}, \operatorname{Pi}\}\{$ Curve $\{c+1\} ; \operatorname{Curve}\{c\} ;\} ;$

$\mathrm{s} 1=\operatorname{ext}[1] ;$

$\mathrm{s} 2=\operatorname{ext}[4] ; \quad 45$

$\mathrm{cl}=$ newll; $\quad 46$

Curve Loop $(\mathrm{cl})=\{\mathrm{c}+1,-(\mathrm{c}+3),-(\mathrm{c}+5), \mathrm{c}\} ; \quad 47$

$\mathrm{s} 3=\mathrm{s} 2+1 ;$

Plane Surface $(\mathrm{s} 3)=\{\mathrm{cl}\} ; \quad 49$

sl=newsl; $\quad 50$

Surface Loop $(\mathrm{sl})=\{\mathrm{s} 1, \mathrm{~s} 2, \mathrm{~s} 3\} ;$

Delete $\{$ Surface $\{8\} ;\} \quad 52$

Volume $(2)=\{\mathrm{sl}\} ;$

BooleanDifference $\{$ Volume $\{2\}$; Delete; $\}\{$ Volume $\{1\}$; Delete; \} 54

Delete $\{$ Surface $\{6\} ;\} \quad 55$

Строки 1-6 задают использование геометрического ядра OpenCascade и параметры затупленного конуса - радиус сферы $R$, длину образующей конуса $L$, угол полураствора конуса alpha и угол атаки, переводя их из градусов в радианы. Строки 7-29 задают затупленный по сфере конус. Плоская проекция конуса состоит из дуги окружности (строка 14), и двух прямолинейных образующих (строки 19 и 21). В строке 22 задается выталкивание (вытягивание) контура конуса по углу на 180 градусов, которое образует искомую половину конуса. При операции выталкивания образуются три поверхности, а плоскость симметрии задается в виде замкнутого цикла ребер (строка 24). Замкнутый цикл из четырех поверхностей задает поверхность затупленного конуса (строка 27), на основе которого создается собственно тело конуса в виде объема 1 (строка 29). Промежуточные элементы геометрии удаляются (строки 15, 28). Вид полученного конуса представлен в левой части рис. 2.

В строке 30 осуществляется поворот конуса на заданный угол атаки. В строках 31-53 задается область обтекания. В стоках 35-38 задаются опорные точки, на основе которых в строке 40 строится сплайн области входа потока. В стоке 42 строится прямолинейная образующая области выхода потока. Аналогично объекту проекция области обтекания выталкивается (вытягивается) по углу и формируется объем 2 (строка 53). Вычитая из объема 2 объем 1 (строка 54), получаем искомую область обтекания вокруг конуса, изображенную в правой части рис. 2. Промежуточные элементы геометрии также удаляются.

Строки 56-59 задают физические поверхности входа потока, выхода потока, плоскости симметрии и поверхности объекта. Под физическими плоскостями (точками, кривыми, объемами) в терминологии пакетов генерации сеток и систем моделирования понимаются геометрические объекты, которые используются для моделирования и/или на которых ставятся граничные условия. В правой части операторов стоит перечень геометрических поверхно- 
стей, которые войдут в физическую поверхность. После формирования сетки данные физические поверхности записаны в файл в качестве граничных поверхностей. Также необходимо определить физический объем, в котором будет производиться моделирование (строка 60).

PhysicalSurface("inlet") = \{8\};

PhysicalSurface("outlet") $=\{7\}$;

PhysicalSurface("symmetry") $=\{9\}$;

PhysicalSurface("wall") = $\{1,2,3\}$;
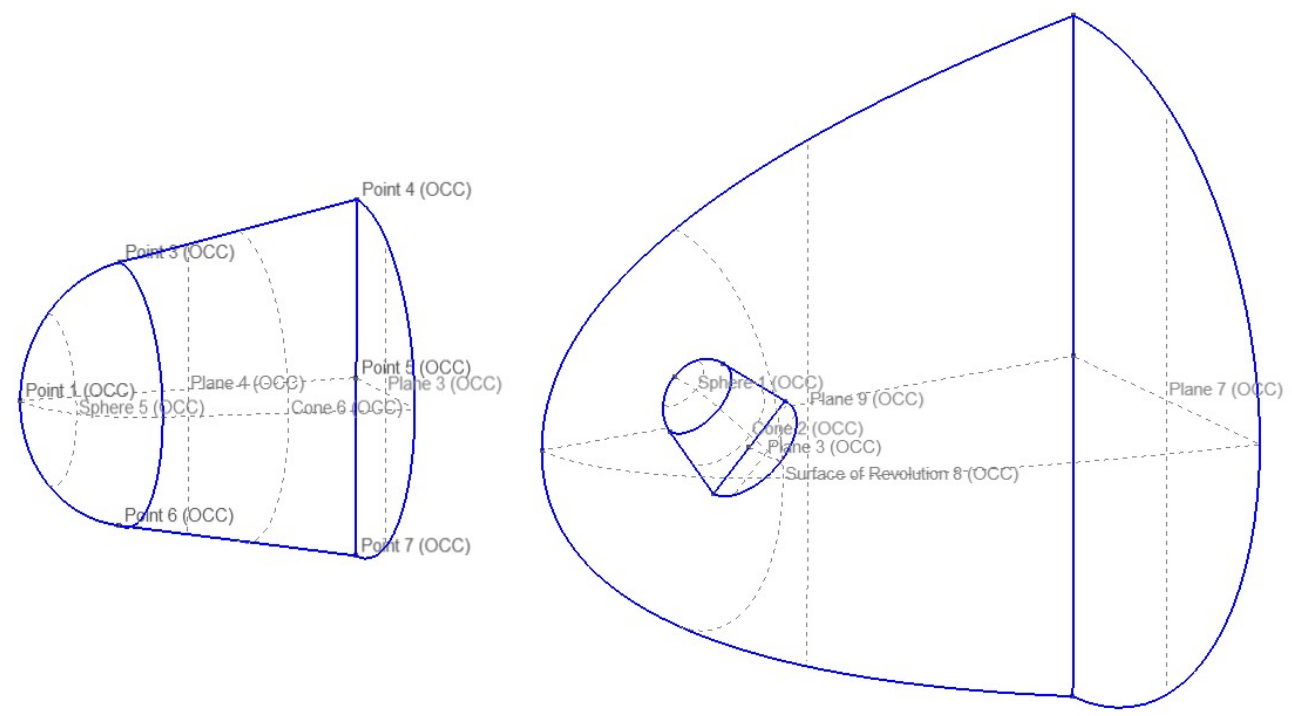

Рис. 2. Вид затупленного конуса (слева) и области обтекания вокруг затупленного конуса под углом атаки (справа)

Остальные операторы (61-74) связаны с генерацией сетки. Характерный размер $h 1$ приписывается всему диапазону точек (строка 63), после чего точкам 7, 9, 11 и 12, лежащим на поверхностях входа и выхода потока, приписывается характерный размер $h 2$ (строка 64). В результате этого характерный размер сетки на обтекаемом конусе будет равен $h l$, а на внешней области обтекания - $h 2$. Остальные операторы задают построение сетки на основе характерных размеров в точках (строки 65,66$)$, число итераций сглаживания двумерной сетки, алгоритм Delaunay для поверхностной сетки и алгоритм НХТ для объемной сетки, включение оптимизации пространственной сетки и параметр оптимизации.

$\mathrm{h} 1=0.013$;

CharacteristicLength $\{:\}=\mathrm{h} 1$;

CharacteristicLength $\{7,9,11,12\}=\mathrm{h} 2$;

Mesh.CharacteristicLengthFromPoints $=1$;

Mesh.CharacteristicLengthExtendFromBoundary $=1$;

Mesh. CharacteristicLengthMin $=\mathrm{h} 1$;

Mesh. CharacteristicLengthMax $=h 2$;

Mesh.Smoothing=2;

General.ExpertMode=1;

Mesh.Algorithm=5;

Mesh.Algorithm3D=10;

// алгоритм hxt 3d

Mesh.Optimize $=1$; 
Приведенный скрипт может быть загружен в интерактивную среду Gmsh (рис. 1) и выполнены команды генерации трехмерной сетки и записи полученной сетки в файл. Того же результата можно добиться вызовом пакета в командной строке:

$>$ gmsh.exe example.geo -3 -o example.neu,

где первый параметр задает скрипт с расширением gео, второй параметр задает генерацию трехмерной сетки, а третий параметр задает выходной файл сетки. Большое количество примеров скриптов с комментариями представлены в документации к пакету [4].

Для построения сеток целесообразно использовать неравномерные сетки со сгущениями в областях наибольших градиентов решения, управляя распределением локального размера ячеек заданием этого размера в вершинах геометрического объекта. Пример использования равномерной сетки и неравномерной сетки с заданием нескольких характерных линейных размеров сетки представлен на рис. 3. Использование неравномерных сеток позволяет существенно уменьшить количество расчетных ячеек, используя более крупные ячейки там, где наличие мелких ячеек не является обязательным. Опыт построения сеток показал, однако, что различие ячеек сетки с отношением линейных размеров более чем 10 является нецелесообразным. Отметим, что при отношении линейных размеров ячеек в 10 раз, объемы таких ячеек различаются в 1000 раз.

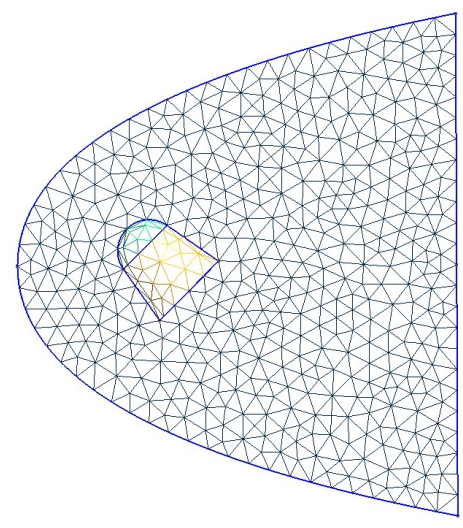

$a$

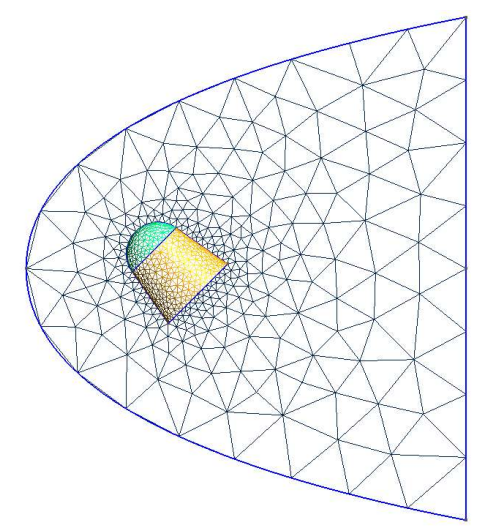

$\sigma$

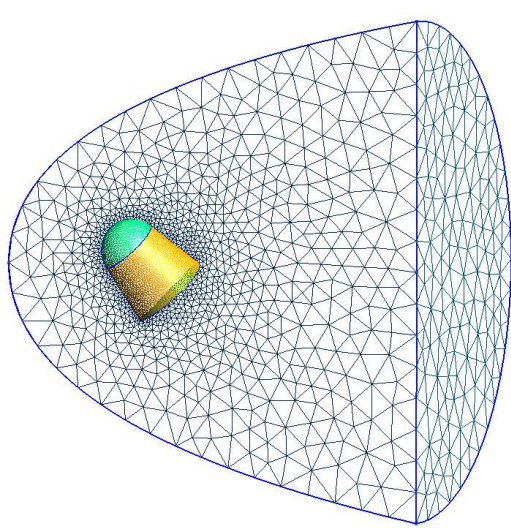

B

Рис. 3. Вид поверхностной сетки на затупленном конусе, в плоскости симметрии и выходной плоскости области для равномерной сетки (a) и двух неравномерных сеток (б) и (b) с различных углов зрения

Для исследования возможностей пакета тестировался весь набор имеющихся в его составе алгоритмов с точки зрения качества получаемой сетки, быстродействия и использования оперативной памяти. Для генерации поверхностной сетки используются алгоритмы MeshAdapt, Delaunay и Frontal-Delaunay. Для сложных поверхностей наиболее предпочтительным считается MeshAdapt, наиболее быстрым - Delaunay и генерирующим наиболее качественную сетку - Frontal-Delaunay. Опыт использования подтвердил, что Delaunay является самым быстрым из них, в то время как другие два алгоритма могли существенно замедляться на отдельных поверхностях.

Для генерации трехмерных сеток используются также три алгоритма: Delaunay, Frontal и HXT. Алгоритм Delaunay считается наиболее безотказным, а алгоритм HXT - наиболее быстрым, являясь новой эффективной реализацией параллельного алгоритма Delaunay.

Оценки производительности наиболее быстрого алгоритма НХТ построения равномерной двумерной и трехмерной сеток в зависимости от линейного размера ячеек представлены в табл. 1 для сеток объемом от 0.9 до 102.9 млн ячеек на ПК Intel i7-9700K.

Время, необходимое только для генерации трехмерной сетки, для сетки размером 3 млн ячеек составляет примерно 10 секунд, в то время как для сетки в 100 млн ячеек время генерации сетки составляет менее 5 минут. Приведенные времена относятся к вычислениям на 
одном ядре процессора. Требуемая оперативная память для генерации этих сеток не превышает 16 ГБ.

\section{Таблица 1}

Объем тетраэдральной сетки и время генерации поверхностной и объемной сетки в зависимости от линейного размера

\begin{tabular}{|c|c|c|c|}
\hline Линейный размер & Сетка, млн ячеек & Время 2d, сек & Время 3d, сек \\
\hline 0.03 & 0.9 & 1.7 & 2.2 \\
\hline 0.02 & 2.8 & 3.8 & 6.2 \\
\hline 0.01 & 22.6 & 16.6 & 45 \\
\hline 0.08 & 44.7 & 27 & 85 \\
\hline 0.06 & 102.9 & 49 & 202 \\
\hline
\end{tabular}

\section{4. Оценка качества генерируемых сеток}

Продолжим сравнение различных методов генерации сеток, дополнив его исследованием качества сгенерированных сеток. Для оценки качества сетки был написан небольшой код. В качестве критерия качества элемента сетки рассматривается параметр качества $q$ [2, 14], равный умноженному на 3 отношению радиуса вписанной в тетраэдр сферы $(r)$ к радиусу описанной сферы $(R)$

$$
q=\frac{3 r}{R}
$$

Этот параметр не превышает единицы для любых тетраэдров, причем значения единицы он достигает для тетраэдра, имеющего все равные между собой ребра и, соответственно, грани. Радиусы вписанной в тетраэдр и описанной сферы вычисляются по формулам

$$
\begin{gathered}
r=\frac{3 V}{S_{1}+S_{2}+S_{3}+S_{4}}, \\
R=\frac{S_{\text {tria }}}{6 V},
\end{gathered}
$$

где $V$ - это объем тетраэдра (1/6 смешанного произведения векторов, исходящих из одной вершины); $S_{1}, S_{2}, S_{3}$ и $S_{4}$ - площади граней тетраэдра; $S_{\text {tria }}$ - площадь треугольника со сторонами $\left(a_{12} a_{34}, a_{13} a_{24}, a_{14} a_{23}\right)$, где $a_{i j}$ - есть длина ребра, соединяющая вершины $i$ и $j$ тетраэдра.

Площадь треугольника $S$ со сторонами $a, b$ и $c$ может быть вычислена по формуле Герона

$$
S^{2}=p(p-a)(p-b)(p-c),
$$

где $p=(a+b+c) / 2$ является половиной периметра треугольника.

Для оценки качества будем оценивать распределение параметра качества $q$ по удельному весу ячеек. Чем больше вес ячеек с высоким качеством, тем больше кривая распределения сдвигается в сторону значения аргумента $q=1$. На рис. 4 представлены распределения качества тетраэдров после генерации равномерной сетки с характерным линейным размером $h=0.068$ всеми тремя методами без оптимизации (слева), генерации сетки методам Delaunay c оптимизацией gmsh и netgen и генерации сетки методом HXT с оптимизацией hxt c параметрами 0.3 и 0.45 (справа). 
Для более детального анализа генерируемых сеток дополнительно введем в рассмотрение значения минимального $q_{\min }$ и среднего $q_{\text {ave }}$ значений параметра качества, а также минимального и максимального радиуса вписанной сферы, нормированного на радиус $r_{h}$ вписанной сферы в правильный тетраэдр с длиной ребра $h$, являющегося характерным размером сетки. Радиус вписанной сферы в правильный тетраэдр с ребром $h$ составляет $\sqrt{6} h / 12$. В табл. 2 представлены значения указанных параметров для сеток без сгущения с параметром $h=0.068$ для различных алгоритмов генерации трехмерной сетки и оптимизации. Размер генерируемой сетки варьируется от 8.6 до 18 млн ячеек. Для всех трех алгоритмов минимальное значение параметра качества $q$ без использования оптимизации мало, а для алгоритма hxt практически равно нулю. Вместе с тем, среднее значение параметра $q$, согласно рис. 4 и таблице 2 остается приемлемым и без оптимизации.
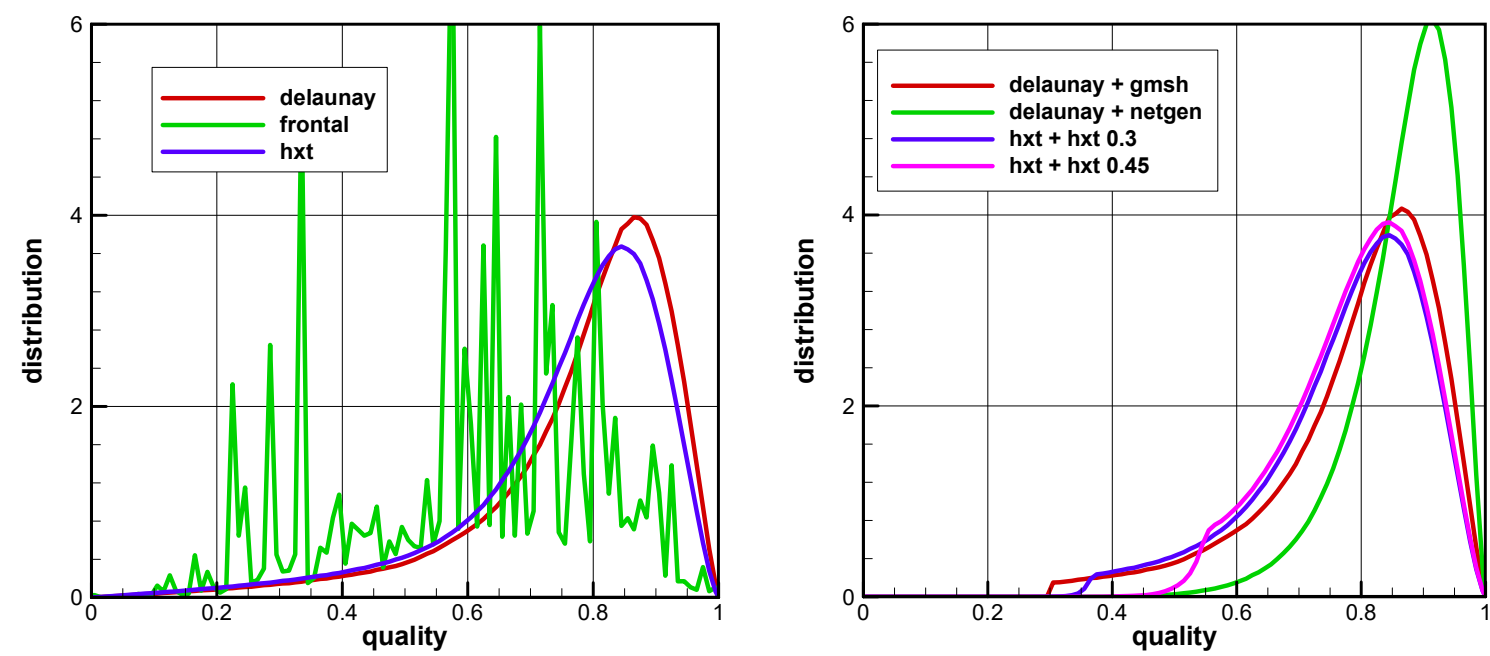

Рис. 4. Распределение функции качества сетки $q$ для алгоритмов без оптимизации (слева) и алгоритмов Delaunay и HXT с оптимизацией (справа)

Как следует из анализа рис. 4, применение оптимизации gmsh подобно эффективному обрезанию распределения качества для значений ниже 0.3 (красная кривая), а применение оптимизации netgen - эффективному обрезанию для значений ниже 0.5 с одновременным сдвигом в сторону высоких $q$ (зеленая кривая). Однако, использование оптимизации netgen требует затрат времени, существенно превышающего времени генерации сетки без оптимизации. Применение оптимизации hxt, доступной только для алгоритма генерации сетки НХТ, подобно эффективному обрезанию распределения параметра $q$ ниже значений 0.3 (синяя кривая) и 0.45 (фиолетовая кривая) при значениях параметра оптимизации 0.3 и 0.45 , соответственно. Однако, оптимизация с параметром 0.45 требует существенно больше времени. В последних колонках таблицы 2 представлены значения минимального и максимального радиуса вписанной сферы нормированного на радиус $r_{h}$.

Глобальный вывод из анализа данных рис. 4 и табл. 2 состоит в том, что комбинация алгоритма генерации трехмерной сетки hxt и одноименной оптимизации строит не менее качественные сетки, чем остальные алгоритмы, в смысле параметра качества ячеек и разброса их размеров. В то же время указанная комбинация более чем на порядок превосходит скорость построения сеток остальными алгоритмами.

Аналогичный вывод можно сделать и из анализа данных табл. 3, в которой представлены характеристики сеток со сгущением, соответствующему характерному размеру сетки на затупленном конусе, равному $h=0.01313$, и характерному размеру сетки на внешней поверхности области обтекания, равному $10 h$. Следует однако отметить, что сетки, построенные с использованием алгоритма frontal и оптимизацией netgen могут быть более качественными, чем построенные с помощью более быстрого алгоритма hxt. 
Таблица 2

Характеристики тетраэдральных сеток без сгущения, полученных по разным алгоритмам генерации сетки и ее оптимизации

\begin{tabular}{|l|l|c|c|l|l|l|l|}
\hline Алгоритм & $\begin{array}{c}\text { Оптими- } \\
\text { зация }\end{array}$ & $\begin{array}{c}\text { Время, } \\
\text { сек }\end{array}$ & $\begin{array}{c}\text { Сетка, } \\
\text { млн ячеек }\end{array}$ & \multicolumn{1}{c|}{$q_{\min }$} & $q_{\text {ave }}$ & $r_{\min } / r_{h}$ & $r_{\max } / r_{h}$ \\
\hline delaunay & & 220 & 11.4 & $0.533 \times 10^{-3}$ & 0.788 & 0.00078 & 1.730 \\
\hline & gmsh & +33 & 11.2 & 0.198 & 0.798 & 0.303 & 1.731 \\
\hline & netgen & +950 & 8.6 & 0.255 & 0.877 & 0.382 & 2.129 \\
\hline frontal & & 468 & 18.0 & $0.344 \times 10^{-8}$ & 0.576 & $0.506 \times 10^{-8}$ & 2.273 \\
\hline & gmsh & +156 & 17.1 & 0.081 & 0.618 & 0.162 & 2.273 \\
\hline & netgen & +945 & 9.6 & 0.117 & 0.862 & 0.269 & 2.044 \\
\hline hxt & & 14.9 & 10.0 & 0 & 0.758 & 0 & 2.100 \\
\hline & hxt 0.3 & +1.4 & 9.7 & 0.219 & 0.776 & 0.405 & 2.100 \\
\hline & hxt 0.45 & +41 & 9.4 & 0.232 & 0.792 & 0.454 & 2.050 \\
\hline
\end{tabular}

Таблица 3

Характеристики тетраэдральных сеток со сгущением, полученных по разным алгоритмам генерации сетки и ее оптимизации

\begin{tabular}{|l|l|c|c|l|l|l|}
\hline Алгоритм & Оптимизация & $\begin{array}{c}\text { Время, } \\
\text { сек }\end{array}$ & $\begin{array}{c}\text { Сетка, } \\
\text { млн ячеек }\end{array}$ & \multicolumn{1}{|c|}{$q_{\min }$} & $q_{\text {ave }}$ & \multicolumn{1}{|c|}{$r_{\min } / r_{h}$} \\
\hline delaunay & & 120 & 6.2 & $0.828 \times 10^{-3}$ & 0.779 & $1.25 \times 10^{-3}$ \\
\hline & gmsh & +16 & 6.1 & 0.197 & 0.791 & 0.350 \\
\hline & netgen & +478 & 4.8 & 0.201 & 0.867 & 0.344 \\
\hline frontal & & 263 & 4.1 & $0.352 \times 10^{-7}$ & 0.609 & $0.992 \times 10^{-6}$ \\
\hline & gmsh & +23.6 & 3.9 & $1.587 \times 10^{-2}$ & 0.641 & 0.065 \\
\hline & netgen & +210 & 2.6 & 0.202 & 0.835 & 0.330 \\
\hline hxt & & 19.8 & 10.0 & 0 & 0.758 & 0 \\
\hline & hxt 0.3 & +1.4 & 9.7 & 0.195 & 0.776 & 0.403 \\
\hline & hxt 0.45 & +39 & 9.4 & 0.206 & 0.791 & 0.499 \\
\hline
\end{tabular}

Система Gmsh также успешно генерирует сетки для обтекания ЛА, характеризуемых сложной геометрией поверхности. Примером такого ЛА является X-43, грубая поверхностная сетка для которого представлена на рис. 1. Успешное построение расчетной сетки возможно при корректном задании геометрии и топологии рассматриваемой модели области и при отсутствии слишком малых геометрических линейных и угловых размеров модели.

\section{5. Примеры расчетов на сгенерированных сетках}

Дополнительно, в том числе для демонстрации корректности построенных тетраэдральных сеток, приведем результаты математического моделирования обтекания конуса, затупленного по сфере, и ЛА Х-33 с использованием вычислительного кода на основе уравнений Эйлера. Моделирование обтекания ЛА является конечным шагом в технологической цепочке: создание геометрии объекта, генерация неструктурированной тетраэдральной сетки, моделирование обтекания объекта.

Моделирование обтекания конуса, закругленного по сфере, проводилось при числе Маха набегающего потока 6.77 при угле атаки 45 градусов на сетке в 2 млн ячеек. Моделиро- 
вание обтекания ЛА X-33 проводилось при числе Маха набегающего потока 6 и нулевом угле атаки на сетке в 30 млн ячеек. Расчет проводился на основе уравнений Эйлера по схеме С.К. Годунова второго порядка точности с использованием локального шага по времени. Расчет проводился до установления «стационарного» режима обтекания.

На рис. 5 представлены распределения давления, отнесенного к величине давления на бесконечности, на поверхности обтекаемого объекта и в плоскости симметрии для обтекания конуса. Распределение давления демонстрирует области повышенного давления в лобовой части ЛА в области его встречи с набегающим потоком и на элементах оперения. В плоскости симметрии обтекания конуса видны скачки уплотнения, волна разрежения на кромке конуса и общая ударно-волновая структура области обтекания. Качественно правильное моделирование обтекания демонстрирует корректное построение пространственной тетраэдральной сетки.
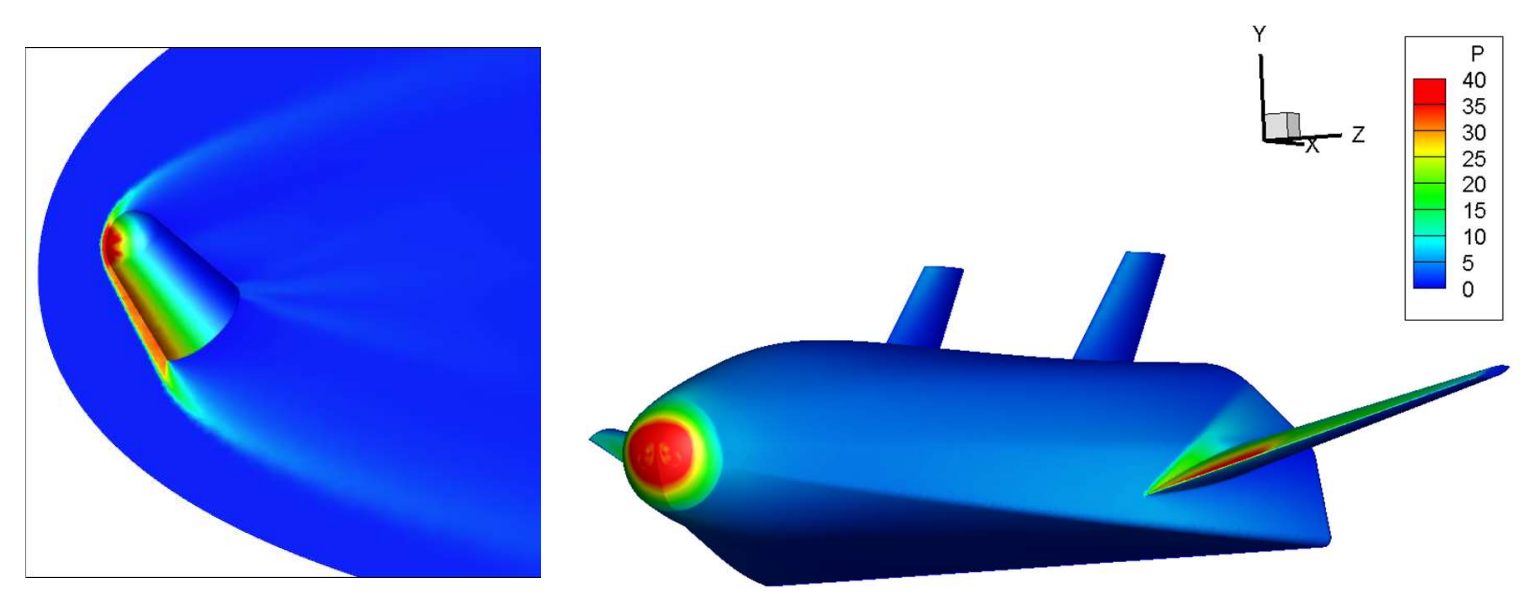

Рис. 5. Распределение давления при обтекании конуса, затупленного по сфере, под углом атаки $45^{\circ}$ при числе Маха 6.77 (слева) и ЛА Х-33 под углом атаки $0^{\circ}$ при числе Маха 6 (справа)

\section{5. Заключение}

Преимуществом использования неструктурированных тетраэдральных сеток является возможность их генерации в полностью автоматическом режиме. Это крайне важно для построения сеток для расчетов обтекания летательных аппаратов и других объектов, обладающих сложной геометрией. В работе рассмотрены два близких по функционалу открытых пакета генерации неструктурированных тетраэдральных сеток - Gmsh и Salome. C помощью пакетов Gmsh и Salome были построены тетраэдральные сетки для большого количества летательных аппаратов, только малая часть из которых представлена в работе. Для пакета Gmsh описаны процедуры создания геометрических объектов и исследованы возможности алгоритмов генерации и оптимизации пространственных сеток с точки зрения качества сетки, минимального размера создаваемых ячеек и быстродействия. Наиболее эффективным оказался новый метод генерации трехмерных сеток НХТ с использованием встроенной в него оптимизации, позволяющий строить неравномерные сетки объемом до 100 млн ячеек за время до 10 минут на высокопроизводительном ПК. Корректность построенных сеток была проверена моделированием обтекания конуса, затупленного по сфере, и ЛА Х-33 при высоких числах Maxa.

Работа выполнена по теме государственного задания (№ госрегистрации АААА-А2012001169001135-5). 


\section{Литература}

1. Василевский Ю.В., Данилов А.А., Липников К.Н., Чугунов В.Н. Автоматизированные технологии построения неструктурированных расчетных сеток. М.: Физматлит, 2016.

2. Geuzaine C., Remacle J.F. Gmsh: a three-dimensional finite element mesh generator with built-in preand post-processing facilities // Int. J. Numer. Methods Engineering. 2009. 79, N 11. 1309-1331.

3. Marot C., Pellerin J., Remacle J.F. One machine, one minute, three billion tetrahedral // Int. J. Numer. Methods Engineering. 2019. 117, N 9. 967-990. https://doi.org/10.1002/nme.5987

4. http://gmsh.info

5. https://www.salome-platform.org/user-section/about

6. http://www.laduga.ru/salome/index.shtml

7. Schöberl J. Netgen, an advancing front $2 \mathrm{~d} / 3 \mathrm{~d}$-mesh generator based on abstract rules // Comput. Visual. Sci. 1997. 1, N 1. 41-52.

8. Si H. (2015). TetGen, a Delaunay-based Tetrahedral Mesh Generator// ACM Trans. on Math. Software. 2015. 41 N 2. 11:1-11:36.

9. Si H. Tetgen, a quality tetrahedral mesh generator and three-dimensional Delaunay triangulator. 2006. https://www-berlin.de/software/tetgen/files/tetrgen-manual.pdf.

10. Котов М.А., Кузенов В.В. Геометрическое моделирование сложных поверхностей гиперзвуковых летательных аппаратов в САПР // Физико-химическая кинетика в газовой динамике. 2012. T. 13, вып. 4. http://chemphys.edu.ru/issues/2012-13-4/articles/372

11. Котов М.А. Геометрическое моделирование поверхностей гиперзвуковых летательных аппаратов X-43 и X-51А // Физико-химическая кинетика в газовой динамике. 2013. Т. 14, вып. 2. http://chemphys.edu.ru/issues/2013-14-2/articles/393

12. Железнякова А.Л. Молекулярно-динамический метод построения неструктурированных сеток в сложных пространственных областях и на криволинейных поверхностях // Физико-химическая кинетика в газовой динамике. 2012. Т. 13, вып. 4. http://chemphys.edu.ru/issues/2012-134/articles $/ 368$

13. Тихонычев П.С. Метод построения пространственных слоистых сеток для задач обтекания летательных аппаратов с изменяемой формой, основанной на электростатической аналогии // Физико-химическая кинетика в газовой динамике. 2016. Т. 17, вып. 3. http://chemphys.edu.ru/issues/2016-17-3/articles/647

14. Суков С.А. Методы генерации тетраэдральных сеток и их программные реализации // Препринт ИПМ им. М.В. Келдыша. 2015. № 23. 22 с.

\section{References}

1. Vasilevskij, Ju. V., Danilov, A. A., Lipnikov, K. N., Chugunov, V. N. Avtomatizirovannye tehnologii postroenija nestrukturirovanny raschetnyh setok (Automated Technologies for Creation of Nonstructured Numerical Meshes), Fismatlit, Moscow, 2016 [in Russian].

2. Geuzaine, C., Remacle, J. F., "Gmsh: a three-dimensional finite element mesh generator with built-in pre- and post-processing facilities," Int. J. Numer. Methods Engineering, Vol. 79, No. 11, 2009, pp. 1309-1331.

3. Marot, C., Pellerin, J., Remacle, J. F., "One machine, one minute, three billion tetrahedral," Int. J. Numer. Methods Engineering, Vol. 117, No. 9, 2019, pp. 967-990. https://doi.org/10.1002/nme.5987

4. http://gmsh.info

5. https://www.salome-platform.org/user-section/about

6. http://www.laduga.ru/salome/index.shtml

7. Schöberl, J., "Netgen, an advancing front $2 \mathrm{~d} / 3 \mathrm{~d}-\mathrm{mesh}$ generator based on abstract rules," Comput. Visual. Sci., Vol. 1, No. 1, 1997, pp. 41-52. 
8. Si, H., "TetGen, a Delaunay-based Tetrahedral Mesh Generator," ACM Trans. on Math. Software, Vol. 41, No. 2, 2015, pp. 11:1-11:36.

9. Si, H., Tetgen, a quality tetrahedral mesh generator and three-dimensional Delaunay triangulator. 2006. https://www-berlin.de/software/tetgen/files/tetrgen-manual.pdf

10. Kotov, M., Kuzenov, V., "Geometric modeling of complex surfaces of hypersonic aircraft in CAD," Physical-Chemical Kinetics in Gas Dynamics, Vol. 13, No. 4, 2012. http://chemphys.edu.ru/issues/2012-13-4/articles/372

11. Kotov, M., "Geometric modeling of surfaces of hypersonic aircraft X-43 and X-51A," Physical-Chemical Kinetics in Gas Dynamics, Vol. 14, No. 2, 2013. http://chemphys.edu.ru/issues/2013-14-2/articles/393

12. Zheleznyakova, A., "Triangular mesh generation by molecular dynamics method for curved surfaces and complex three-dimensional domains," Physical-Chemical Kinetics in Gas Dynamics, Vol. 13, No. 4, 2012. http://chemphys.edu.ru/issues/2012-13-4/articles/368

13. Tikhonychev, P. S., "Method for volume layered grid generation for problems of hypersonic flows around vehicles with changing shapes as a result of ablation based on electrostatic analogy," PhysicalChemical Kinetics in Gas Dynamics, Vol. 17, No. 3, 2016. http://chemphys.edu.ru/issues/2016-173/articles/647

14. Sukov, S. A., Metody generacii tetrajedral'nyh setok $i$ ih programmnye realizacii (Tetrahedral mesh generation algorithms and applications), Preprint IPM im. M.V. Keldysha, No. 23, Moscow, 2015.

Статья поступила в редакцию 5 сентября 2020 г. 